\title{
Cardiovascular risk of smoking and benefits of smoking cessation
}

\author{
Giuseppina Gallucci ${ }^{1}$, Alfredo Tartarone ${ }^{2}$, Rosa Lerose ${ }^{3}$, Anna Vittoria Lalinga ${ }^{4}$, Alba Maria Capobianco ${ }^{2}$ \\ ${ }^{1}$ Cardiology Unit, ${ }^{2}$ Department of Onco-Hematology, Division of Medical Oncology, ${ }^{3}$ Hospital Pharmacy, ${ }^{4}$ Pathology Unit, IRCCS-CROB Referral \\ Cancer Center of Basilicata, Rionero in Vulture (PZ), Italy \\ Contributions: (I) Conception and design: G Gallucci, A Tartarone; (II) Administrative support: None; (III) Provision of study materials or patients: \\ None; (IV) Collection and assembly of data: None; (V) Data analysis and interpretation: None; (VI) Manuscript writing: All authors; (VII) Final \\ approval of manuscript: All authors. \\ Correspondence to: Dr. Alfredo Tartarone. Department of Onco-Hematology, Division of Medical Oncology, IRCCS-CROB Referral Cancer Center \\ of Basilicata via Padre Pio 1, 85028 Rionero in Vulture (PZ), Italy. Email: tarta1@virgilio.it.
}

\begin{abstract}
Smoking increases mortality from all causes and has a crucial role in atherosclerotic cardiovascular disease (ASCVD). Active smoking and secondhand smoke exposure determine more than 30\% of coronary heart disease (CHD) mortality. The exact mechanisms of cardiovascular damages are not well known, but the detrimental effect of smoking on endothelial function has long been recognized. Smoking elicits oxidative processes, negatively affects platelet function, fibrinolysis, inflammation and vasomotor function; all these proatherogenic effects double the 10-year risk of fatal events in smokers compared to non smokers. An intriguing issue about smoking is the vulnerability of female gender. The mortality from cardiovascular diseases (CVDs) is higher in female than male smokers and female smokers show a $25 \%$ higher risk of developing CHD than men with the same exposure to tobacco smoke. This female vulnerability seems to be related to genes involved in thrombin signaling. The effects of smoking cessation have also been extensively studied. Cessation at an early age (40 years) has an impressive $90 \%$ reduction in the excess risk of death. In this review we report recent data about the causal link between smoking and CVDs and about the benefits of smoking cessation.
\end{abstract}

Keywords: Cardiovascular diseases (CVDs); tobacco smoking; secondhand smoke

Submitted Feb 05, 2020. Accepted for publication Feb 28, 2020.

doi: $10.21037 /$ jtd.2020.02.47

View this article at: http://dx.doi.org/10.21037/jtd.2020.02.47

\section{Introduction: the causal link}

Cardiovascular diseases (CVDs) are a major cause of morbidity and mortality throughout the world. In the United States, CVDs affect many racial or ethnic groups, and this fact has an extremely high cost that is estimated around $\$ 200$ billion annually in healthcare services, drugs, and loss of productivity. Much of this burden is due to insufficient implementation of prevention strategies and poor control of atherosclerotic cardiovascular disease (ASCVD) risk factors in many adults $(1,2)$. According to World Health Organization data, smoking determines $10 \%$ of all CVDs (3). Tobacco smoking usage causes approximately 6 million death per year throughout the world, in the United States almost 500,000 deaths can be attributed to smoking and about $10 \%$ of these deaths are caused from second-hand smoke exposure. Epidemiologic studies have supported the assumption that cigarette smoking increases the incidence of myocardial infarction and fatal coronary artery diseases (4). The increased risk of cardiovascular events has also been shown for low-tar cigarettes and smokeless tobacco. Even passive smoking is responsible for a $30 \%$ increased risk of ASCVD, a little less than half of the risk increase in active smokers that is around $80 \%(5,6)$. Ever since the Framingham study, the epidemiologic investigations have tried to identify people with a high likelihood for a future cardiovascular events in order to make actionable interventions to reduce the risk. The concept of "risk factors" was made popular by Kannel et 
Table 1 Chronic diseases causally linked to smoking and secondhand smoke exposure according to Surgeon General's Report 2010-2014 (5,6)

\begin{tabular}{|c|c|c|c|}
\hline \multicolumn{2}{|l|}{ Smoking } & \multicolumn{2}{|l|}{ Secondhand smoke exposure } \\
\hline Stroke & Stroke & $\mathrm{CHD}$ & $\mathrm{CHD}$ \\
\hline Aortic aneurysm & Aortic aneurysm & Reproductive effects in women & Reproductive effects in women \\
\hline $\mathrm{CHD}$ & $\mathrm{CHD}$ & Nasal irritation & Nasal irritation \\
\hline COPD & COPD & & \\
\hline Pneumonia & Pneumonia & & \\
\hline Cataracts & Cataracts & & \\
\hline \multirow[t]{4}{*}{ Reproductive effects in women } & Reproductive effects in women & & \\
\hline & Erectile dysfunction & & \\
\hline & Rheumatoid arthritis & & \\
\hline & Immune function & & \\
\hline
\end{tabular}

CHD, coronary heart diseases; APAD, atherosclerotic peripheral artery disease; COPD, chronic obstructive pulmonary disease.

$a l$. with their publication "Factors of risk in the development of Coronary Heart disease" (7). In that era, the first clinical risk scores were popularized, a logistic model with 7 risk factors was proposed and it included "cigarettes smoked" (8). The Framingham Risk score for coronary heart disease (CHD), published in 1998 by Wilson and colleagues, is the most popular risk profile; sex-specific prediction equations were formulated to predict CVD risk according to age, diabetes, smoking, blood pressure categories, total cholesterol and LDL cholesterol categories (9). This score has been adopted by the Adult Treatment Panel of the National Cholesterol Education Program (NCEP) in the United States (10). In 2014 (6), 50 years after the first report, new causal conclusions for active and secondhand smoke were added, not included in 2010 (5) (Table 1). The 2014 report made these statements about active smoking and cardiovascular diseases: "there is sufficient evidence to derive a causal relationship between a smoke free policy and a reduction in coronary events in a population younger than 65 years of age" (6). In 2016 the Report emphasized the potential damage of the extensive use of e-cigarette among young adults and stressed the importance of urgent actions to protect the health of this young population (11).

Smoking tobacco is causally related to many phenotypes of CVDs, it is linked to early onset atherosclerosis that starts in adolescents and young adults, increases the risk of acute myocardial infarction (AMI), stroke, peripheral artery disease, aortic aneurysm and sudden death $(4-6,12,13)$.

As far as secondhand smoke is concerned, several components of passive smoking (e.g., carbonyls and particulate matter) have demonstrated important toxic cardiovascular effects (6). It has also been shown by Frey et al. (14) that even a small period of exposure to secondhand smoke (30 minutes) in a place where smoking is permitted, has a detrimental effect on endothelial function with an increased risk for acute coronary events and hospitalizations. More than 20 individual-level studies have shown an association between passive smoking and risk of stroke, meta-analyses have documented a $20-30 \%$ increased risk of stroke with a likely doseresponse relationship (15). In the recent 2019 ACC/AHA Prevention Guidelines cigarette smoking has been defined a "strong, independent risk factor for CVD events and premature death" $(16,17)$. In the National Health Interview Survey, ASCVD in young people was causally related to smoking (18).

\section{Smoking and cardiovascular diseases}

While the link of smoking and CVD is substantiated by 
epidemiologic studies, the precise mechanisms responsible for this association have not been defined yet. Cigarette smoking is the most puzzling and complex risk factor for CVDs because it is a mixture of chemical compounds that are either bound to aerosol particles or free in the gas phase. It has been estimated that cigarette smoke has over 7,000 chemical compounds from many different classes, including at least 72 carcinogens (19-21). Fowles et al. in 2003 have tried to link chemical compounds to toxicity, and they studied the risk for chronic disease, they associated 1,3-butadiene to cancer risk, and cyanide arsenic and the cresols to cardiovascular risk (22).

A few American smokers know that ammonia, arsenic, lead, mercury and radioactive elements are contained in cigarettes, most of them know only about carbon monoxide and nicotine. What is even worse is the scanty awareness of the public on the unhealthy effects of smoking, for example, $60 \%$ of people do not know that smoking can cause impotence (23-25).

Nicotiana tabacum plants are able to accumulate metals and this ability is utilized to decontaminate soil from metals. But this ability becomes dangerous when toxic metals like aluminium, chromium, copper, lead, mercury, nickel, and zinc accumulate in tobacco products like filters and cigarette smoke. Recent data suggest that cigarette smoking alters metal homeostasis and this effect may induce chronic diseases (26). Bernhard and colleagues. showed that metals contained in cigarette smoke play a crucial role in damaging the vascular endothelium $(27,28)$. They can catalyze processes that lead to oxidative stress and, consequently, to damage and inflammation, the "root" causes of non-communicable chronic diseases such as cardiovascular diseases, cancers, degenerative diseases, and aging. Polycyclic aromatic hydrocarbons delivered by cigarette smoking are ligands for the aryl hydrocarbon receptor (AhR). Smoking up-regulates the expression of inflammatory genes, too. Another agonist of the AhR, 2,3,7,8-tetrachlorodibenzo-p-dioxin (TCDD), accelerates the atherosclerotic process in apoliproprotein E-deficient mice (29). Moreover, the accumulation of cholesterol within macrophages in vitro is stimulated by cigarette smoke extract; a relevant role in this process is played by the CXCR2 chemotactic receptor that is considered a pivotal element in inflammatory disease including atherosclerosis (30). In 1992 Celermajer et al. showed that active continuous smoking impairs Flow Mediated Dilatation (FMD) of the brachial artery and that this effect is dose-dependent (31). Reduction of endothelial dependent dilatation was also found in coronary arteries and was reversible (32). A significant reversal of FMD after one year of smoking cessation was indeed reported by Johnson et al. (33). A study compared the effect of light and regular cigarettes on endothelial function, the Authors found that the effect of chronic smoking on endothelial function is independent of the type of cigarette smoked and that even the lowest dose of cigarettes impairs FMD (34). Secondhand smoking, too, negatively affects FMD and this effect can be reversed 1 year after cessation of exposure (35). In 2004 the Surgeon General's report listed six pathogenetic mechanisms of smoking-induced heart disease: (I) endothelial damage, (II) prothrombotic effect, (III) inflammation, (IV) abnormal lipid metabolism, (V) increased myocardial oxygen and blood demand and (VI) decreased myocardial blood and oxygen supply (36). Nicotine effects seem to be induced by stimulation of the nicotinic acetylcholine receptors (nAChRs), located in the Central Nervous System and in other organs in the body that are component of the parasympathetic autonomic nervous system. The increased cardiovascular risk seems to be related to the adrenergic effects of nicotine that result in an increased heart rate, increased inotropic status, increased coronary microvascular resistance and reduced insulin sensitivity (5).

But it is important to focus on the main target of the toxic effects of smoking: the endothelium, a "multitask" organ with anti-inflammatory, antithrombotic and vasomotor properties that regulate and maintain vascular tone and haemostasis. Smoking affects all these important properties and the ensuing endothelial "dysfunction" is the first step of the atherogenic process (37).

One phenotype of endothelial dysfunction is the impairment of the endothelium-dependent vasodilatation, a process that needs nitric oxide. Both active and passive smokers have impaired vasodilatation that seems to be worse in passive smokers (38). The reduced nitric oxide bioavailability in smokers is mainly due to the free radicals in the gaseous phase of smoking that contains superoxide anions (39). Many studies in the literature come to the conclusion that oxidant compounds of cigarette smoke cause endothelial dysfunction which is the primum movens of the atherosclerotic process and that the plaques of smokers have a peculiar vulnerability due to a higher extracellular lipid content, an important involvement of inflammatory cells, and a high propensity to haemorrhage in the inner portion of the plaque. Another player in the vulnerability of the atheromatous plaque is the increased activity of matrix metalloproteinase elicited by smoking-induced oxidative 
stress (40-43).

\section{Smoking and thrombogenicity}

A dysfunctioning endothelium loses its antithrombotic properties, but smoking itself influences platelet aggregability. The balance between proaggregatory thromboxane A2 produced by platelets and antiaggregatory prostacyclin secreted by vascular cells has been studied in habitual smoker; it has been shown that smoking induces proaggregatory conditions thus leading to a hypercoagulable state. Smoking elicits an increased generation of von Willebrand factor, and prothrombotic factors, while impairing the process of fibrinolysis (44-46). The activation of platelet and of the coagulation cascade, with a reduction of fibrinolysis in smokers have been confirmed in more recent studies. Sudden cardiac deaths and AMI are frequently caused by acute rupture of a coronary atheromatous plaque with acute coronary artery thrombosis. Cigarette smoking is the leading factor for acute coronary thrombosis, as a consequence sudden cardiac death induced by acute thrombosis are frequent in cigarette smokers (4). The link between smoking and acute thrombosis has been documented in both active and passive cigarette smoking. Barua et al. examined in vitro the effects of smoke exposure on clot and fibrin architecture, using thromboelastography, GP IIb/IIa inhibition and electron microscopy. They showed that acute cigarette smoke exposure is associated with shortening of the time for fibrin formation and augmented clot strength, these two mechanisms can explain heightened thrombogenicity of the atheromatous plaques of smokers (47).

\section{The "lipid effect"}

Besides the direct effect on endothelial function, smoking has an effect on serum lipids that can enhance endothelial damage. Craig and colleagues. showed that smoking increases, in a statistically significant way, total cholesterol, very-low and low- density lipoprotein, and triglyceride serum levels. The Authors also found decreased concentrations of high density lipoprotein (HDL) and apolipoprotein A1 in smokers (48). Other subsequent clinical studies documented a proatherogenic modification of serum lipid profiles induced by smoking $(49,50)$. Moreover, smoking induces lipid oxidation, oxidatively modified LDLs are captured by macrophages that become foam cells, thus initiating the process of plaque formation (51-53).
An interesting observation has been made by Neufeld and colleagues. in children with a high propensity for early onset heart disease (due to a genetic form of dyslipidemia), the Authors found in these children a significant reduced levels of HDL after secondhand smoke in the household, this effect did not show the reversibility with smoking cessation that has been described with the impaired FMD (54). In a population of $\sim 3,000$ healthy women (30-70 years) passive smoking was linked to negative effects on glucose and lipid profiles, increasing the risk of diabetes and cardiovascular disease (55).

\section{The "diabetic effect"}

A large body of literature have suggested that smoking increases the risk of type 2 diabetes mellitus (T2DM) with pathogenetic studies supporting the liaison between smoking and T2DM (56-63). The first data of a link between smoking and diabetes are from early 90's of last century $(64,65)$, however, T2DM is multifactorial in etiology and the molecular mechanisms of increased incidence of both insulin resistance and T2DM in smokers are not well defined, yet. Smoking has a detrimental effect of endothelial function, increases inflammation/oxidative stress and directly damage $\beta$-cell function. Tobacco products contain thousands of chemical compounds and many free radicals that may be responsible of the pathogenetic process that leads to smoke-related insulin resistance or diabetes. In the British Regional Heart Study, active cigarette smokers were confronted with people who never smoked, current cigarette smokers had a higher risk of diabetes, even after adjustments for age, BMI, and other potential confounders (66). Other studies have linked smoking to insulin resistance (67-69). Smoking can affect insulin sensitivity also through epigenetic mechanisms: smokinginduced diabetes susceptibility may be due to aberrant methylation of DNA (70). Moreover, smoking has an impact on pancreatic $\beta$ cell function, but the mechanisms are less well known.

\section{Smoking and blood pressure}

Whereas the influence of smoking on lipid levels and insulin resistance is well documented, data on the effect of smoking on blood pressure are conflicting. In 2010 Virdis et al. stated that cigarette smoking has an acute hypertensive effect mediated by the stimulation of the sympathetic nervous system. For chronic smoking available data do 
not prove that smoking directly induces hypertension, and smoking cessation does not lead to a reduction of blood pressure values, either. Nevertheless, the effect of smoking on arterial stiffness may have a greater impact on central blood pressure that is related to target organ damage more closely if compared to brachial blood pressure (71). In a more recent study Saladini et al. investigated the effect of smoking on peripheral and central blood pressure in a group of young stage I hypertensives (72). Central systolic blood pressure and pulse pressure were higher in smokers than in nonsmokers, thus implying a predominant effect on central blood pressure (72).

\section{Smoking and the immune system}

Another intriguing issue is the link between smoking and the immune system. Smoking activates the immune system systemically and locally, increasing white blood cell counts and the level of neutrophils, lymphocytes and monocytes. Increased levels of proinflammatory cytokines are also found in smokers, along with increased level of C-reactive protein. The immunologic alterations induced by smoking affect local inflammatory processes in the vascular wall with increased expression of matrix metalloproteinase, leukocyte recruitment and increased plasma concentration of soluble vascular cell adhesion molecules (73-75). The impact of smoking on immune function is stressed in the 2014 Surgeon General's Report: (I) there is sufficient evidence that components of cigarette smoke affect components of the immune system, with either an activating or suppressing effect; (II) there is sufficient evidence that cigarette smoking has a damaging effect on the immune system that increases the risk of pulmonary infections; (III) there is sufficient evidence that cigarette smoke has a detrimental effect of immunocompetence and this effect increases the risk of immune-mediated diseases. In the same report it is said that "there is sufficient evidence of a causal link between cigarette smoking and rheumatoid arthritis and of a reduced effectiveness of the tumor necrosis factor-alpha (TNF- $\alpha$ ) inhibitors" (6). Qiu et al. in a more recent paper review the influence of smoking on major components of innate and adaptive immune cells (76).

We now know that chronic inflammatory conditions such as rheumatoid arthritis and other autoimmune disorders are risk enhancers for cardiovascular diseases (16) and that there are increasing data that relate a state of low-grade inflammation both to stable cardiovascular disease and to cancer, therefore it can be speculated that the effects of smoking on immune system does have an impact on CVDs.

\section{Cerebral blood flow}

Cigarette smoking has an impact on cerebral blood flow too, increasing the risk of stroke and the severity of stroke. Smoking impairs nitric oxide (NO)-mediated flow increase by reducing $\mathrm{NO}$ synthesis in cerebrovascular endothelial cells, thus interfering with blood flow and glucose metabolism in the cerebral circulation. In the brain, as in other vascular bed, the endothelial damage is the "primum movens" of the effect. (NO)-mediated vasodilation is reduced through inhibition of nitric oxide synthase of the endothelium (eNOS) and of the neurons (nNOS) and by the overproduction of oxygen radicals. Nicotine has an acute and chronic effect on the eNOS and negatively affect nitrergic nerve function. These effects induce the synthesis of amyloid beta that speeds up the reduction of blood flow and may be causally related to Alzheimer disease (77). For passive smoking there are many studies that prove a causal relationship between secondhand smoke and increased risk of stroke as documented in the 2014 Surgeon General' s Report (6).

\section{Erectile dysfunction (ED)}

Cigarette use in an independent risk factor for ED. The association between chronic smoking and ED is well documented but the mechanisms of cigarette-induced ED are not completely understood. In an in vivo rat model of chronic cigarette-induced ED it was shown that oxidative stress was significantly higher in the exposed group compared to control animal. Mean neuronal NOS expression was significantly lower and apoptotic index significantly higher in cigarette smoke exposed animals compared to control animals (78).

\section{Smoking cessation}

Smoking is a recognized risk factor for ASCVD and determines 20\% of CVD deaths in the United States, therefore smoking cessation is strongly recommended to reduce the CVD burden. But the time course of cardiovascular risk reduction following smoking cessation have not been defined, when we compare smokers to never smokers. Many cardiovascular risk calculators do not make differences between people who smoked for some years of their life and people who never smoked. The ASCVD 
Risk Estimator Plus make a distinction between smokers and never smokers but states that cardiovascular risk of "ex-smokers" becomes identical to the risk of people who never smoked after 5 years (79). But this statement is not well proven and the time course of cardiovascular risk reduction following smoking cessation is not well defined. This uncertainty may lead to an underestimation of the risk among former smokers. In the National Health Interview Survey, smoking was causally related to ASCVD even in young people (18), and this is the reason why the main recommendation is abstinence from a very early age. Nevertheless, smoking cessation is still beneficial in reducing excess risk even among older adults (80). In a recent study, Duncan et al. evaluated the relationship between years since smoking cessation and events of CVD retrospectively analyzing prospectively collected data from Framingham Heart Study population who did not have CVD at baseline and were followed up through December 2015. The study population included 8,770 people $(3,805$ from the first cohort examined for the fourth time in 1954-1958 and 4,965 from the offspring cohort examined for the first time in 1971-1975). Heavy smokers ( $\geq 20$ pack-years) who quit smoking showed a lower risk of CVD within 5 years compared to current heavy smokers and this reduction was significant (hazard ratio 0.61), confirming the cardiovascular benefit of smoking cessation observed in other studies. The study also showed a very slow CVD risk reduction over time. Former heavy smokers ceased to be significantly associated with an increased CVD risk only after 10 to 15 years following smoking cessation, when compared to never smokers $(5-10$ years in the first recruited cohort and $\geq 25$ years in the offspring cohort). Including former smokers with $<20$ pack-years their excess CVD risk ceased to be significant within 10 to 15 years of cessation, if compared to never smokers. This observation stresses the importance of stratifying the risk by cumulative pack-years to avoid underestimation of the risk after 5 years of quitting in former heavy smokers (81).

In a very recent paper Delgado et al. (82) studied the influence of smoking and quitting smoking on biomarkers of endothelial function and their association with mortality. The goal of the study was to analyze the impact of smoking on circulating markers of endothelial dysfunction and to investigate whether such effects have an influence on the potential use of these markers to estimate cardiovascular risk. In the study, smokers showed higher concentration of soluble InterCellularAdhesionMolecule-1 (sICAM-1), of sE-selectin expressed by endothelial cells and leukocytes and of sP-selectin expressed by endothelial cells, and concentration of sL-selectin expressed on leucocytes and soluble VascularCellAdhesionMolecule-1 (sVCAM-1). Levels of sL-selectin were reduced even after 20 years of smoking abstinence and were inversely correlated to mortality in active smoker improving risk prediction.

\section{Gender issue}

The risk of smoking-related chronic diseases in women has showed a sharp rise in the last 50 years and, for CVD, the risk has reached the numbers observed in men. Cigarette smoking seems to be more harmful to women. In 2002 the Surgeon General published the second Report on Women and smoking (83), the first was published in 1980 (84). Some of the main topics were: (I) smoking is a leading cause of CHD in women. For women younger than 50 years, smoking is the cause of the majority of CHD. The number of cigarettes smoked and the duration of smoking increase the risk; (II) the risk for CHD in women can be reduced significantly 1 or 2 years after smoking cessation; (III) women who smoke and use oral contraceptives have a very high risk of CHD; (IV) the risk for ischemic stroke and subarachnoid hemorrhage is increased in women who smoke; (V) smoking affect progression and severity of carotid atherosclerotic lesions in women. The rate of progression of carotid atherosclerosis can be slowed by smoking cessation; (VI) the risk of peripheral arterial disease is increased in women who smoke; (VII) the risk of death from ruptured abdominal aortic aneurysm is increased in woman who smoke. In 2011 Huxley and Woodward estimated the effect of smoking on CHD in women compared with men reviewing 8,005 abstracts and included 26 articles with data for 3,912,809 individuals, in 75 cohorts (2.4 million participants) adjusted for cardiovascular risk factors other than CHD. The authors reported a pooled adjusted female-to-male Relative Risk Ratio (RRR) of smoking compared to no smoking for CHD of 1.25. The pathogenetic mechanisms that determine these differences are not defined yet, they could be biological (e.g., thrombin signaling) or related to differences in smoking behavior between men and women (85).

In 2013 Campesi et al. (86) enrolled healthy adult men and women with regular menstrual cycles not taking oral contraceptives. In women cigarette smoking reduced DNA methylation more if compared to men, moreover an increased number of platelets, monocytes 
and lymphocytests and increased levels of homocysteine, arginine, and ADMA were observed only in women. An increased number of neutrophil and eosinophil was shown only in men. Smoking has the highest negative impact in young females. The Pooling Project on Diet and Coronary Heart Disease (87) reported for women 40-49 years of age who smoke a hazard ratio of 8.5 compared to never smokers, whereas the hazard ratio decreased to 3.1 in women aged 70 years or older.

It seems that cardiovascular risk factors come earlier in young healthy female smokers if compared to young healthy male smokers, and this fact should support the need for more studies to better understand the sex \& gender differences in smoking effects. In addition, women have peculiar smoking related risks linked to pregnancy and to the use of oral contraceptives. For all these reasons, cessation programs targeting women are urgently needed.

\section{Conclusions}

Cigarette smoking is the number one cause of diseases that can be effectively prevented. The smoking-induced CVD risk is primarily determined by the value of Pack-years. It has also been proved that smoking fewer cigarettes/ day for longer duration is more dangerous than smoking more cigarettes/day for shorter duration (88). All these observations strengthen the need of smoking cessation rather the smoking reduction. Cigarette smoking is not only a risk factor for chronic CVD, but it is also an inducer of acute atherothrombotic events such as stroke or myocardial infarction ad has an impact on glucose tolerance and levels of HDL cholesterol. The role of inflammation in non-communicable diseases is an emergent issue and smoking elicits a proinflammatory state. A recent study documented that low-grade inflammation is a risk factor for incident lung cancer in patients with stable CVD, another epiphenomenon of the shared risk factors between CVD and cancer (89). Smoking cessation has a great role in reducing the cardiovascular risk, but for heavy smokers there is still a remarkable residual risk after 5 year that should not be underestimated (81). The war against tobacco still needs commitments by all health stakeholders because the best weapon is the absolute abstinence from smoking and to achieve this important result it is important to intervene at a population level with a battle against all unhealthy lifestyle behaviors. The American Heart Association define ideal cardiovascular health as "The absence of clinically manifest CVD together with the presence of the 7 metrics that compose Life's Simple 7: not smoking, having a healthy diet pattern, adequate physical activity, healthy body weight, healthy blood pressure, cholesterol and blood glucose in the absence of pharmacological treatment. Therefore, to achieve cardiovascular health we should focus on children, adolescents, young adults and young families to ensure healthy habits early in life and sustained throughout the life course (90).

\section{Acknowledgments}

Funding: None.

\section{Footnote}

Provenance and Peer Review: This article was commissioned by the Guest Editor (Alfredo Tartarone) for the Series "Improving Outcomes in Lung Cancer Through Early Diagnosis and Smoking Cessation" published in fournal of Thoracic Disease. The article was sent for external peer review organized by the Guest Editor and the editorial office.

Conflicts of Interest: All authors have completed the ICMJE uniform disclosure form (available at http://dx.doi. org/10.21037/jtd.2020.02.47). The series "Improving Outcomes in Lung Cancer Through Early Diagnosis and Smoking Cessation" was commissioned by the editorial office without any funding or sponsorship. AT served as the unpaid Guest Editor of the series and serves as an unpaid editorial board member of Journal of Thoracic Disease from Aug 2019 to Jul 2021. The authors have no other conflicts of interest to declare.

Ethical Statement: The authors are accountable for all aspects of the work in ensuring that questions related to the accuracy or integrity of any part of the work are appropriately investigated and resolved.

Open Access Statement: This is an Open Access article distributed in accordance with the Creative Commons Attribution-NonCommercial-NoDerivs 4.0 International License (CC BY-NC-ND 4.0), which permits the noncommercial replication and distribution of the article with the strict proviso that no changes or edits are made and the original work is properly cited (including links to both the formal publication through the relevant DOI and the license). See: https://creativecommons.org/licenses/by-nc-nd/4.0/. 


\section{References}

1. Weir HK, Anderson RN, Coleman King SM, et al. Heart disease and cancer deaths-trends and projections in the United States, 1969-2020. Prev Chronic Dis 2016;13:E157.

2. Johnson NB, Hayes LD, Brown K, et al. CDC National Health Report: leading causes of morbidity and mortality and associated behavioral risk and protective factorsUnited States, 2005-2013. MMWR Suppl 2014;63:3-27.

3. WHO global report: mortality attributable to tobacco 2012.

4. Barua RS, Ambrose JA Mechanisms of coronary thrombosis in cigarette smoke exposure Arterioscler Thromb Vasc Biol 2013;33:1460-67.

5. Centers for Disease Control and Prevention (US); National Center for Chronic Disease Prevention and Health Promotion (US); Office on Smoking and Health (US). How tobacco smoke causes disease: the biology and behavioral basis for smoking-attributable disease: a report of the Surgeon General. Atlanta (GA): Centers for Disease Control and Prevention (US); 2010.

6. National Center for Chronic Disease Prevention and Health Promotion (US) Office on Smoking and Health. The Health Consequences of Smoking-50 Years of Progress: A Report of the Surgeon General. Atlanta (GA): Centers for Disease Control and Prevention (US); 2014.

7. Kannel WB, Dawber TR, Kagan A, et al. Factors of risk in the development of coronary heart disease--six year follow-up experience. The Framingham Study. Ann Intern Med 1961;55:33-50.

8. Truett J, Cornfield J, Kannel W. A multivariate analysis of the risk of coronary heart disease in Framingham. J Chronic Dis 1967;20:511-24.

9. Wilson PW, D'Agostino RB, Levy D, et al. Prediction of coronary heart disease using risk factor categories. Circulation 1998;97:1837-47.

10. Lipsy RJ The National Cholesterol Education Program Adult Treatment Panel III guidelines. J Manag Care Pharm 2003;9:2-5

11. National Center for Chronic Disease Prevention and Health Promotion (US) Office on Smoking and Health. E-cigarette Use among Youth and Young Adults: A Report of the Surgeon General. Atlanta (GA). Centers for Disease Control and Prevention (US); 2016.

12. Benowitz NL. Cigarette smoking and cardiovascular disease: pathophysiology and implications for treatment. Prog Cardiovasc Dis 2003;46:91-111

13. Csordas A, Wick G, Laufer G et al. An evaluation of the clinical evidence on the role of inflammation and oxidative stress in smoking-mediated cardiovascular disease.

Biomark Insights 2008;3:127-39.

14. Frey PF, Ganz P, Hsue PY, et al. The exposure-dependent effects of aged secondhand smoke on endothelial function. J Am Coll Cardiol 2012;59:1908-13.

15. Oono IP, Mackay DF, Pell JP. Meta-analysis of the association between secondhand smoke exposure and stroke. J Public Health (Oxf) 2011;33:496-502.

16. Arnett DK, Blumenthal RS, Albert MA et al. ACC/AHA Guideline on the Primary Prevention of Cardiovascular Disease: Executive Summary: A Report of the American College of Cardiology/American Heart Association Task Force on Clinical Practice Guidelines. J Am Coll Cardiol 2019;74:1376-414.

17. Pan A, Wang $\mathrm{Y}$, Talaei $\mathrm{M}$, et al. Relation of smoking with total mortality and cardiovascular events among patients with diabetes mellitus: a meta-analysis and systematic review. Circulation 2015;132:1795-804.

18. Khan RJ, Stewart CP, Davis SK, et al. The risk and burden of smoking related heart disease mortality among young people in the United States. Tob Induc Dis 2015;13:16.

19. Burns DM Cigarettes and cigarette smoking. Clin Chest Med 1991;12:631-42.

20. Rodgman A, Perfetti TA. The chemical components of tobacco and tobacco smoke; CRC Press, 2008.

21. Rodgman A, Smith CJ, Perfetti TA. The composition of cigarette smoke: a retrospective, with emphasis on polycyclic components. Hum Exp Toxicol 2000;19:573-95.

22. Fowles J, Dybing E. Application of toxicological risk assessment principles to the chemical constituents of cigarette smoke. Tob Control 2003;12:424-30.

23. Cummings KM, Hyland A, Giovino GA et al. Are smokers adequately informed about the health risks of smoking and medicinal nicotine? Nicotine Tob Res 2004;6 Suppl $3:$ S333-40.

24. Ramo DE, Delucchi KL, Liu H et al.Young adults who smoke cigarettes and marijuana: analysis of thoughts and behaviors. Addict Behav 2014;39:77-84.

25. Gravely S, Fong GT, Cummings KM, et al. Awareness, trial, and current use of electronic cigarettes in 10 countries: Findings from the ITC project. Int J Environ Res. Public Health 2014;11:11691-704.

26. Messner B, Bernhard D. Smoking and cardiovascular disease Arterioscler Thromb Vasc Biol 2014;34:509-15.

27. Bernhard D, Rossmann A, Wick G. Critical Review Metals in Cigarette Smoke IUBMB Life 2005;57:805-9.

28. Bernhard D, Csordas A, Henderson B, et al. Cigarette 
smoke metal-catalyzed protein oxidation leads to vascular endothelial cell contraction by depolymerization of microtubules. FASEB J 2005;19:1096-107.

29. Wu D, Nishimura N, Kuo V, et al. Activation of aryl hydrocarbon receptor induces vascular inflammation and promotes atherosclerosis in apolipoprotein E-/- mice. Arterioscler Thromb Vasc Biol 2011;31:1260-7.

30. Boisvert WA, Santiago R, Curtiss LK, et al. A leukocyte homologue of the IL-8 receptor CXCR-2 mediates the accumulation of macrophages in atherosclerotic lesions of LDL receptor-deficient mice. J Clin Invest 1998;101:353-63.

31. Celermajer DS, Sorensen KE, Gooch VM, et al. Non-invasive detection of endothelial dysfunction in children and adults at risk of atherosclerosis. Lancet 1992;340:1111-5.

32. Zeiher AM, Schächinger V, Minners J. Long-term cigarette smoking impairs endothelium-dependent coronary arterial vasodilator function. Circulation 1995;92:1094-100.

33. Johnson HM, Gossett LK, Piper ME, et al. Effects of smoking and smoking cessation on endothelial function: 1-year outcomes from a randomized clinical trial. J Am Coll Cardiol 2010;55:1988-95.

34. Amato M, Frigerio B. Castelnuovo S, et al. Effects of smoking regular or light cigarettes on brachial artery flowmediated dilation. Atherosclerosis 2013;228:153-60.

35. Otsuka R, Watanabe H, Hirata K, et al. Acute effects of passive smoking on the coronary circulation in healthy young adults. JAMA 2001;286:436-41.

36. Office of the Surgeon General (US); Office on Smoking and Health (US). The Health Consequences of Smoking: A Report of the Surgeon General. Atlanta (GA): Centers for Disease Control and Prevention (US); 2004.

37. Fuster V, Moreno PR, Fayad ZA, et al. Atherothrombosis and high-risk plaque: part I: evolving concepts. J Am Coll Cardiol 2005;46:937-54.

38. Celermajer DS, Adams MR, Clarkson P, et al. Passive smoking and impaired endothelium-dependent arterial dilatation in healthy young adults. $\mathrm{N}$ Engl J Med 1996;334:150-4.

39. Barua RS, Ambrose JA. Reactive oxygen species are involved in smoking-induced dysfunction of nitric oxide biosynthesis and upregulation of endothelial nitric oxide synthase: an in vitro demonstration in human coronary artery endothelial cells. Circulation 2003;107:2342-7.

40. Shah PK, Falk E, Badimon JJ, et al. Human monocytederived macrophages induce collagen breakdown in fibrous caps of atherosclerotic plaques. Potential role of matrix- degrading metalloproteinases and implications for plaque rupture. Circulation 1995;92:1565-9.

41. Newby AC. Metalloproteinases and vulnerable atherosclerotic plaques. Trends Cardiovasc Med 2007;17:253-8.

42. Zhang $\mathrm{K}$, Huang XZ, Li XN, et al. Interleukin 6 destabilizes atherosclerotic plaques by downregulating prolyl-4-hydroxylase $\alpha 1$ via a mitogen-activated protein kinase and c-Jun pathway. Arch Biochem Biophys 2012;528:127-33.

43. Csordas A, Bernhard D. The biology behind the atherothrombotic effects of cigarette smoke. Nat Rev Cardiol 2013;10:219-30.

44. Nielsen VG, Hafner DT, Steinbrenner EB. Tobacco smoke-induced hypercoagulation in human plasma: role of carbon monoxide. Blood Coagul Fibrinolysis 2013;24:405-10.

45. Sambola A, Osende J, Hathcock J, et al. Role of risk factors in the modulation for tissue factor activity and blood thrombogenicity. Circulation 2003;107:973-7.

46. Newby DE, McLeod AL, Uren NG, et al. Impaired coronary tissue plasminogen activator release is associated with coronary atherosclerosis and cigarette smoking: direct link between endothelial dysfunction and atherothrombosis. Circulation 2001;103:1936-41.

47. Barua RS, Sy F, Srikanth S, et al. Effects pf Cigarette Smoke Exposure on Clot Dynamics and Fibrin Structure. Arterioscler Thromb Vasc Biol 2010;30:75-9.

48. Craig WY, Palomaki GE, Haddow JE. Cigarette smoking and serum lipid and lipoprotein concentrations: an analysis of published data. BMJ 1989;298:784-8.

49. Freedman DS, Srinivasan SR, Shear CL, et al. Cigarette smoking initiation and longitudinal changes in serum lipids and lipoproteins in early adulthood: the Bogalusa Heart Study. Am J Epidemiol 1986;124:207-19.

50. Nakamura K, Barzi F, Huxley R, et al. Pacific Cohort Studies Collaboration. Does cigarette smoking exacerbate the effect of total cholesterol and high-density lipoprotein cholesterol on the risk of cardiovascular diseases? Heart 2009;95:909-16.

51. Reilly M, Delanty N, Lawson JA, et al. Modulation of oxidant stress in vivo in chronic cigarette smokers. Circulation 1996;94:19-25.

52. Pilz H, Oguogho A, Chehne F, et al. Quitting cigarette smoking results in a fast improvement of in vivo oxidation injury (determined via plasma, serum and urinary isoprostane). Thromb Res 2000;99:209-21.

53. Solak ZA, Kabaroğlu C, Cok G, et al. Effect of different 
levels of cigarette smoking on lipid peroxidation, glutathione enzymes and paraoxonase 1 activity in healthy people. Clin Exp Med 2005;5:99-105.

54. Neufeld EJ, Mietus-Snyder M, Beiser AS, et al. Passive cigarette smoking and reduced HDL cholesterol levels in children with high-risk lipid profiles. Circulation 1997;96:1403-7.

55. Gu L, Li J, Pan G, et al. Effects of passive smoking on glycemic parameters and lipid profiles in a Chinese female population. Clin Lab 2017;63:1147-52.

56. Will JC, Galuska DA, Ford ES, et al. Cigarette smoking and diabetes mellitus: evidence of a positive association from a large prospective cohort study. Int J Epidemiol 2001;30:540-6.

57. Foy CG, Bell RA, Farmer DF, et al. Smoking and incidence of diabetes among U.S. adults: findings from the Insulin Resistance Atherosclerosis Study. Diabetes Care 2005;28:2501-7.

58. Willi C, Bodenmann P, Ghali WA, et al. Active smoking and the risk of type 2 diabetes: a systematic review and meta-analysis. JAMA 2007;298:2654-64.

59. Xie XT, Liu Q, Wu J, et al. Impact of cigarette smoking in type 2 diabetes development Acta Pharmacologica Sinica 2009;30:784-7.

60. Sliwińska-Mossoń M, Sciskalska M, Karczewska-Gorska P, et al. The effect of endothelin-1 on pancreatic diseases in patients who smoke. Adv Clin Exp Med 2013;22:745-52.

61. Śliwińska-Mossoń M, Milnerowicz H. The impact of smoking on the development of diabetes and its complications. Diab Vasc Dis Res 2017;14:265-76.

62. Maddatu J, Anderson-Baucu, E, Evans-Molina C. Smoking and the risk of Type 2 Diabetes Transl Res 2017;184:101-7.

63. Campagna D, Alamo A, Di Pino A, et al. Smoking and diabetes: dangerous liaisons and confusing relationships. Diabetol Metab Syndr 2019;11:85.

64. Rimm EB, Manson JE, Stampfer MJ, et al. Cigarette smoking and the risk of diabetes in women. Am J Public Health 1993;83:211-4.

65. Rimm EB, Chan J, Stampfer M, et al. Prospective study of cigarette smoking, alcohol use, and the risk of diabetes in men. BMJ 1995;310:555-9.

66. Wannamethee SG, Shaper AG, Perry IJ. British Regional Heart Study. Smoking as a modifiable risk factor for type 2 diabetes in middle-aged men. Diabetes Care 2001;24:1590-5.

67. Facchini FS, Hollenbeck CB, Jeppesen J, et al. Insulin resistance and cigarette smoking. Lancet 1992;339:1128-30.

68. Attvall S, Fowelin J, Lager I, et al. Smoking: a potential link with the insulin resistance syndrome. J Intern Med 1993;233:327-32.

69. Houston TK, Person SD, Pletcher MJ, et al. Active and passive smoking and development of glucose intolerance among young adults in a prospective cohort: CARDIA study. BMJ 2006;332:1064-9.

70. Besingi $W$, Johansson A. Smoke-related DNA methylation changes in the etiology of human disease. Hum Mol Genet 2014;23:2290-7.

71. Virdis A, Giannarelli C, Neves MF, et al. Cigarette smoking and hypertension. Curr Pharm Des 2010;16:2518-25.

72. Saladini F, Benetti E, Fania C, et al. Effects of smoking on central blood pressure and pressure amplification in hypertension of the young. Vasc Med 2016;21:422-8.

73. Scott DA, Palmer RM. The influence of tobacco smoking on adhesion molecule profiles. Tob Induc Dis 2002;1:7-25.

74. Sopori M. Effects of cigarette smoke on the immune system. Nat Rev Immunol 2002;2:372-7.

75. Stämpfli MR, Anderson GP. How cigarette smoke skews immune responses to promote infection, lung disease and cancer. Nat Rev Immunol 2009;9:377-84.

76. Qiu F, Liang CL, Liu H, et al. Impacts of cigarette smoking on immune responsiveness: $\mathrm{Up}$ and down or upside down? Oncotarget 2017;8:268-84.

77. Toda N, Okamura T. Cigarette smoking impairs nitric oxide-mediated cerebral blood flow increase: Implications for Alzheimer's disease. J Pharmacol Sci 2016;131:223-32.

78. Huang YC, Chin CC, Chen CS, et al. Chronic Cigarette Smoking Impairs Erectile Function through Increased Oxidative Stress and Apoptosis, Decreased nNOS, Endothelial and Smooth Muscle Contents in a Rat Model. PLoS One 2015;10:e0140728.

79. Lloyd-Jones DM, Huffman MD, Karmali KN, et al. Estimating longitudinal risks and benefits from cardiovascular preventive therapies among medicare patients: the Million Hearts longitudinal ASCVD risk assessment tool: a special report from the American Heart Association and American College of Cardiology. Circulation 2017;135:e793-813.

80. Mons U, Müezzinler A, Gellert C, et al. Impact of smoking and smoking cessation on cardiovascular events and mortality among older adults: meta-analysis of individual participant data from prospective cohort studies of the CHANCES consortium. BMJ 2015;350:h1551.

81. Duncan MS, Freiberg MS, Greevy RA, et al. Association of smoking cessation with subsequent risk of cardiovascular disease. JAMA 2019;322:642-50. 
82. Delgado GE, Kramer BK, Siekmeier R, et al. Influence of smoking and smoking cessation on biomarkers of endothelial function and their association with mortality. Atherosclerosis 2020;292:52-9.

83. Women and smoking. A Report of the Surgeon General, 2002.

84. Women and smoking. A Report of the Surgeon General, 1980.

85. Huxley RR, Woodward M. Cigarette smoking as a risk factor for coronary heart disease in women compared with men: a systematic review and meta-analysis of prospective cohort studies. Lancet 2011;378:1297-305.

86. Campesi I, Carru C, Zinellu A, et al. Regular cigarette smoking influences the transsulfuration pathway, endothelial function, and inflammation biomarkers in a

Cite this article as: Gallucci G, Tartarone A, Lerose R, Lalinga AV, Capobianco AM. Cardiovascular risk of smoking and benefits of smoking cessation. J Thorac Dis 2020;12(7):38663876. doi: $10.21037 /$ jtd.2020.02.47 sex-gender specific manner in healthy young humans. Am J Transl Res 2013;5:497-509.

87. Tolstrup JS, Hvidtfeldt UA, Flachs EM, et al. Smoking and risk of coronary heart disease in younger, middle-aged, and older adults. Am J Public Health 2014;104:96-102.

88. Lubin JH, Couper D, Lutsey PL, et al. Risk of cardiovascular disease from cumulative cigarette use and the impact of smoking intensity. Epidemiology 2016;27:395-404.

89. Van't Klooster CC, Ridker PM, Hjortnaes J, et al. The relation between systemic inflammation and incident cancer in patients with stable cardiovascular disease: a cohort study. Eur Heart J 2019;40:3901-9.

90. Sanchez E. Life's Simple 7: Vital But Not Easy. J Am Heart Assoc 2018. doi: 10.1161/JAHA.118.009324. 